\title{
Optimum On-line Reduction of Switching Losses with a New Two-Phase PWM Method
}

\author{
Toshiji Kato*a) $^{*}$ Senior Member, Kaoru Inoue* ${ }^{*} \quad$ Member \\ Kyu Takano* Non-member, Kazunari Yamada* Non-member \\ Ariyasu Aki* Student Member
}

(Manuscript received Dec. 3, 2013, revised March 3, 2014)

\begin{abstract}
This paper presents an efficient pulse-width modulation (PWM) method to optimally reduce switching (SW) losses in three-phase neutral-point clamped (NPC) inverters in one switching cycle using a new two-phase modulation scheme. The proposed method digitally selects an optimum switching vector (SV) pattern, which minimizes an estimation function based on switching losses considering phase currents among possible reduction patterns for one switching cycle. The proposed modulation method is further extended to reduce common-mode voltages in three-phase NPC inverters. The basic principle involves elimination of the common-mode voltage by limiting the combinations of the output phase voltage levels of the three-level NPC inverter. A modified space vector generation technique based on the two-phase modulation method is proposed to reduce both common-mode voltages and switching losses. The proposed PWM method was experimentally verified for output voltages of the NPC inverter connected to a universal load. The method reduced the number of switching transitions for the largest or second largest current, and it can automatically adapt to different load conditions. The method was also experimentally validated for its EMC and switching loss reduction capability.
\end{abstract}

Keywords: three-phase NPC inverter, reduction of switching losses, space vector modulation, two-phase modulation, state feedback control, EMC

\section{Introduction}

Sinusoidal pulse-width modulation (PWM) inverters are widely used; therefore, their switching ( $\mathrm{SW}$ ) losses must be reduced. When current amplitudes of switches are the same, their SW losses are almost proportional to the number of switching transitions. Therefore, techniques for reducing the number of switching transitions, such as the two-phase modulation method ${ }^{(1)-(4)}$, are effective because they generate equivalent PWM waveforms with fewer switching transitions than conventional PWM techniques. In fact, the amplitudes of the three phase currents are different, and an SW pattern (SP) which avoids switching of large phase currents, effectively reduces the $\mathrm{SW} \operatorname{loss}{ }^{(5)-8)}$. This study proposes a novel two-phase modulation method for selecting the optimal SP, which minimizes an estimated SW loss by reducing the switching transitions, considering phase currents among multiple reduction patterns ${ }^{(9)}$.

The proposed SW loss estimation includes two types of SW losses. The first is the total PWM SW loss composed of three switching vectors (SVs) for one switch cycle with the two-phase modulation method. The second is the transition loss due to the transition of one SP to another including switch mode (SM) changes. The proposed SW loss

a) Correspondence to: Toshiji Kato. E-mail: tkato@mail.doshisha. ac.jp

* Department of Electrical Engineering, Doshisha University

1-3, Tatara-Miyakodani, Kyotanabe, Kyoto 610-0321, Japan estimation is defined as the difference between the first loss saving and the second loss. The optimal SP is defined as that with the lowest loss estimation, and it can most effectively reduce SW losses.

The proposed modulation method is extended to reduce common-mode voltages in three-phase neutral-point clamped (NPC) inverters. The basic principle is to eliminate the common-mode voltage by limiting the combinations of output phase voltage levels of the three-level NPC inverter ${ }^{(10)-(13)}$. A modified space vector generation technique based on the two-phase modulation method is proposed to reduce both common-mode voltages and switching losses.

The proposed optimum PWM principle is experimentally verified with a DSP-based digital control system ${ }^{(14)}$. The PWM output voltages and currents of the three-level NPC inverter connected to a universal load are investigated for online optimal selection of an SP, which effectively reduces its SW loss, among possible SP candidates. The method is also experimentally validated for its EMC and switching loss reduction capability.

\section{Reduction of SW Losses Considering Current Amplitudes}

2.1 Two-Phase PWM Method Based on the Space Vector Concept The control of a three-phase three-level NPC inverter shown in Fig. 1 involves of 6 switching modes (SMs) from I to VI, as shown in Fig. 2, depending on the output phases. It has $3^{3}=27 \mathrm{SVs}$ from $V_{0}-V_{26}$, as shown in 


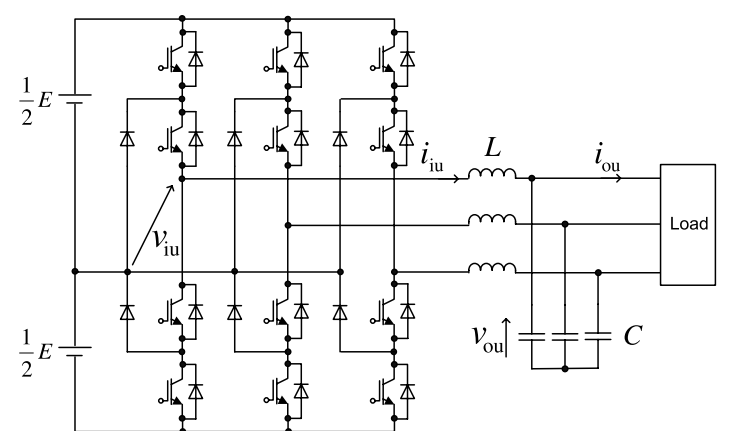

Fig. 1. Three-phase three-level NPC inverter with an LC filter

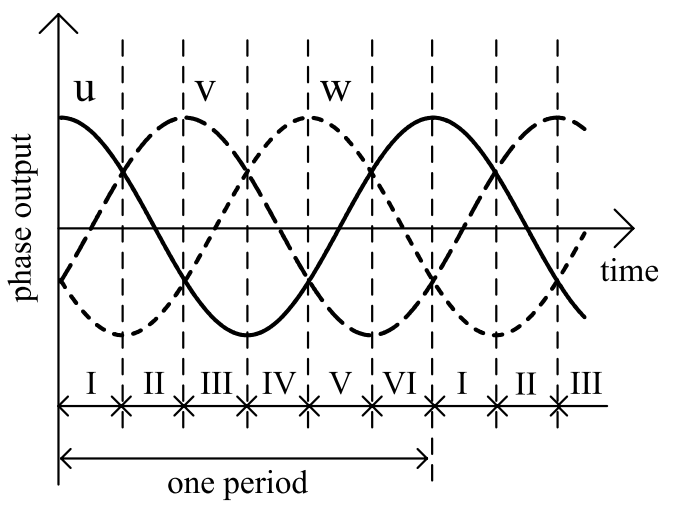

Fig. 2. Three-phase sinusoidal waveforms and their switching mode (SM)

Fig. 3(a), where 1, 1/2, and 0 represent the states in which the upper-arm, middle neutral-arm, and lower-arm switches are on for each phase, $u, v, w$.

To generate sinusoidal waveforms in the three-phases, a reference vector is controlled to trace a circle from SMs I to IV inside the hexagon shown in Fig. 3(a). For example, SVs for SM I are shown in Fig. 3(b) to explain its four minor modes, SM Ia-Id, The situation for the other SMs is the same. The minor modes are determined from a given reference vector value. For example, when the reference is a small value, $\mathrm{SM}$ a is selected, when it is large, SM b, c, or d is selected.

In conventional PWM methods including the triangular waveform comparison method, one switching cycle consists of four SVs. In this study, the two-phase modulation method is adopted because its switching cycle consists of only three SVs that correspond to three vertex vectors of the SM.

For example, when the reference vector is in SM Id, the possible vector combinations are $V_{16}, V_{2}, V_{8}$ or $V_{16}, V_{8}, V_{2}$ as shown in Fig. 4. However, the number of switching transitions depends on the selected SP. When the $V_{16}, V_{2}, V_{8}$ SP is selected, a total of four switchings transitions are required; two switching transitions of phases $u$ and $v$ from $V_{16}$ to $V_{2}$, one switching transition of phase $v$ from $V_{2}$ to $V_{8}$, and one switching of phase $v$ from $V_{8}$ to $V_{16}$, as shown in Fig. 4(a). On the contrary, when the $V_{16}, V_{8}, V_{2} \mathrm{SP}$, or the $V_{2}, V_{8}, V_{16}$ SP is selected for return, as in Fig. 4(b), only two switching transitions are necessary for one switching cycle and the transition from $V_{16}$ to $V_{2}$ can be avoided.

Transitions from one SV to another SV need additional switching transitions because of the differences between the

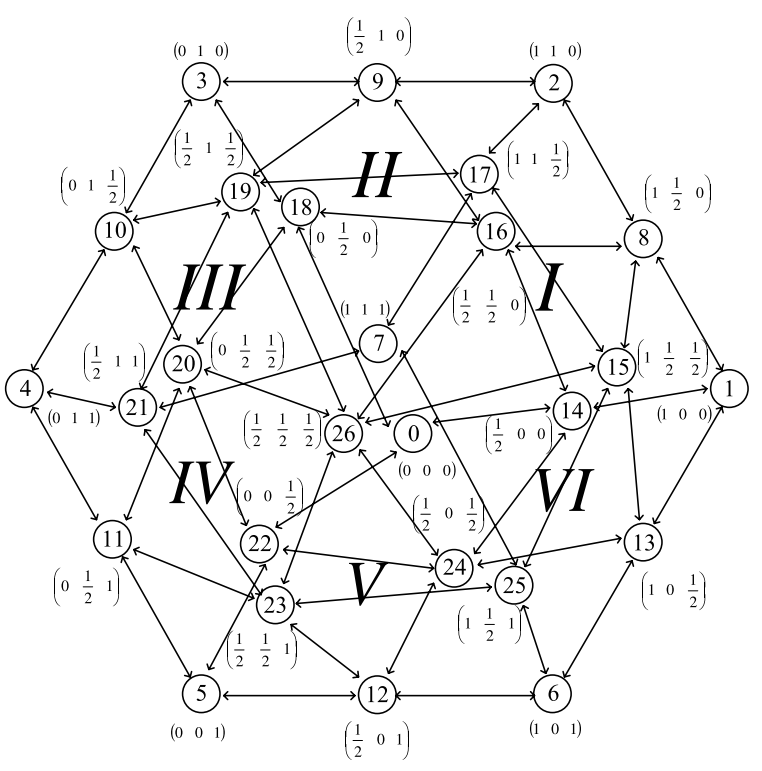

(a) Whole rules for modes I(a)-VI(d)

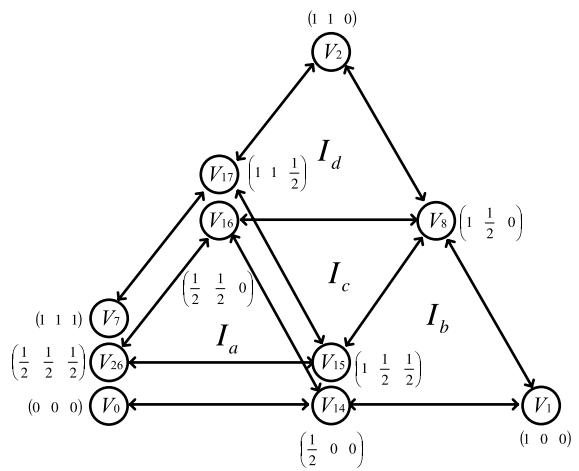

(b) Rules for modes Ia-Id

Fig. 3. Transition rule between switch vectors for the three-level NPC inverter

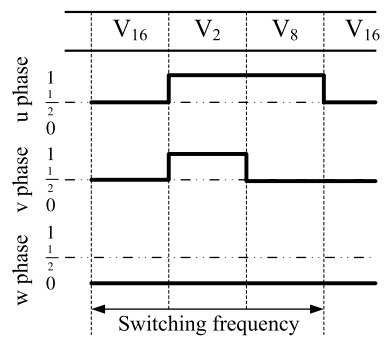

(a) Four switching transitions

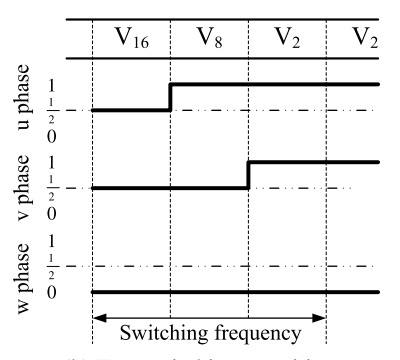

(b) Two switching transitions
Fig. 4. Switch timing reduction using two-phase modulation

two SVs. At least one switching is necessary for any transition. There are 54 transitions, represented by arrows in Fig. 4(a), that satisfy one switching condition. The proposed method utilizes this switching rule to generate sinusoidal waveforms in the three-phases using the two-phase modulation method.

2.2 SW Loss Reduction with the Proposed Two-Phase Modulation Method The proposed PWM method modifies the conventional two-phase modulation technique, and its basic rule is that switching transitions between SVs are only limited to arrow directions as shown in Fig. 3(a) where only one phase is switched along any arrow. Its SPs are shown in 
Table 1. Switch vector combinations for one switching cycle and their loss-saving phases without switching for three-level NPC inverter

\begin{tabular}{|c|c|c|c|c|c|c|c|c|c|c|c|}
\hline I-VI & a-d & $V_{a}$ & $V_{b}$ & $V_{c}$ & saving & I-VI & a-d & $V_{a}$ & $V_{b}$ & $V_{c}$ & saving \\
\hline \multirow[t]{24}{*}{ I } & \multirow[t]{10}{*}{$\mathrm{a}$} & $V_{0}$ & $V_{14}$ & $V_{16}$ & $\mathrm{w}^{*}$ & \multirow[t]{24}{*}{ II } & \multirow[t]{10}{*}{$\mathrm{a}$} & $V_{0}$ & $V_{18}$ & $V_{16}$ & $\mathrm{w}^{*}$ \\
\hline & & $V_{7}$ & $V_{17}$ & $V_{15}$ & $\mathrm{u} *$ & & & $V_{7}$ & $V_{17}$ & $V_{19}$ & $\mathrm{v} *$ \\
\hline & & $V_{14}$ & $V_{16}$ & $V_{26}$ & $\mathrm{u}$ & & & $V_{16}$ & $V_{18}$ & $V_{0}$ & $\mathrm{w} *$ \\
\hline & & $V_{15}$ & $V_{17}$ & $V_{7}$ & $\mathrm{u} *$ & & & $V_{16}$ & $V_{26}$ & $V_{19}$ & $\mathrm{u}$ \\
\hline & & $V_{15}$ & $V_{26}$ & $V_{16}$ & $\mathrm{v}$ & & & $V_{17}$ & $V_{19}$ & $V_{26}$ & w \\
\hline & & $V_{16}$ & $V_{14}$ & $V_{0}$ & $\mathrm{w} *$ & & & $V_{18}$ & $V_{16}$ & $V_{26}$ & $\mathrm{v}$ \\
\hline & & $V_{16}$ & $V_{26}$ & $V_{15}$ & $\mathrm{v}$ & & & $V_{19}$ & $V_{17}$ & $V_{7}$ & $\mathrm{v} *$ \\
\hline & & $V_{17}$ & $V_{15}$ & $V_{26}$ & w & & & $V_{19}$ & $V_{26}$ & $V_{16}$ & $\mathrm{u}$ \\
\hline & & $V_{26}$ & $V_{15}$ & $V_{17}$ & w & & & $V_{26}$ & $V_{16}$ & $V_{18}$ & $\mathrm{v}$ \\
\hline & & $V_{26}$ & $V_{16}$ & $V_{14}$ & $\mathrm{u}$ & & & $V_{26}$ & $V_{19}$ & $V_{17}$ & $\mathrm{w}$ \\
\hline & \multirow[t]{4}{*}{$\mathrm{b}$} & $V_{1}$ & $V_{8}$ & $V_{15}$ & $\mathrm{u}$ & & \multirow[t]{4}{*}{$\mathrm{b}$} & $V_{2}$ & $V_{9}$ & $\overline{V_{16}}$ & $\mathrm{~W}$ \\
\hline & & $V_{8}$ & $V_{1}$ & $V_{14}$ & w & & & $V_{9}$ & $V_{2}$ & $V_{17}$ & $\mathrm{v}$ \\
\hline & & $V_{14}$ & $V_{1}$ & $V_{8}$ & $\mathrm{w}$ & & & $V_{16}$ & $V_{9}$ & $V_{2}$ & w \\
\hline & & $V_{15}$ & $V_{8}$ & $V_{1}$ & $\mathrm{u}$ & & & $V_{17}$ & $V_{2}$ & $V_{9}$ & $\mathrm{v}$ \\
\hline & \multirow[t]{6}{*}{$\mathrm{c}$} & $V_{8}$ & $V_{15}$ & $V_{17}$ & $\mathrm{u}$ & & \multirow[t]{6}{*}{$\mathrm{c}$} & $V_{9}$ & $V_{16}$ & $V_{18}$ & $\mathrm{~W}$ \\
\hline & & $V_{8}$ & $V_{16}$ & $V_{14}$ & $\mathrm{w}$ & & & $V_{9}$ & $V_{19}$ & $V_{17}$ & $\mathrm{v}$ \\
\hline & & $V_{14}$ & $V_{16}$ & $V_{8}$ & $\mathrm{w}$ & & & $V_{16}$ & $V_{9}$ & $V_{19}$ & $\mathrm{u}$ \\
\hline & & $V_{15}$ & $V_{8}$ & $V_{16}$ & $\mathrm{v}$ & & & $V_{17}$ & $V_{19}$ & $V_{9}$ & $\mathrm{v}$ \\
\hline & & $V_{16}$ & $V_{8}$ & $V_{15}$ & $\mathrm{v}$ & & & $V_{18}$ & $V_{16}$ & $V_{9}$ & w \\
\hline & & $V_{17}$ & $V_{15}$ & $V_{8}$ & $\mathrm{u}$ & & & $V_{19}$ & $V_{9}$ & $V_{16}$ & $\mathrm{u}$ \\
\hline & \multirow[t]{4}{*}{$\bar{d}$} & $V_{2}$ & $V_{8}$ & $\overline{V_{16}}$ & $\overline{\mathrm{W}}$ & & \multirow[t]{4}{*}{$\bar{d}$} & $V_{3}$ & $V_{9}$ & $\overline{V_{19}}$ & $\mathrm{~V}$ \\
\hline & & $V_{8}$ & $V_{2}$ & $V_{17}$ & $\mathrm{u}$ & & & $V_{9}$ & $V_{3}$ & $V_{18}$ & w \\
\hline & & $V_{16}$ & $V_{8}$ & $V_{2}$ & w & & & $V_{18}$ & $V_{3}$ & $V_{9}$ & w \\
\hline & & $V_{17}$ & $V_{2}$ & $V_{8}$ & $\mathrm{u}$ & & & $V_{19}$ & $V_{9}$ & $V_{3}$ & $\mathrm{v}$ \\
\hline
\end{tabular}

Table 1. There are ten, four, six, and four SPs for SMs Ia-Id respectively. For example, for SM Ic, the SPs start from $V_{8}$, $V_{14}, V_{15}, V_{16}, V_{17}$. The SP $V_{8}-V_{15}-V_{17}$ can save an $\mathrm{SW}$ loss of phase $u$. The representation is similar for another SP, and an SW loss of one selected phase is reduced. In SM a, the SPs denoted with $*$ are not utilized to simplify operations. However, this limitation has minimal effect because any phase can be selected for reducing loss.

The two phase currents $i_{i u}, i_{i w}$, are measured, and $i_{i v}$ is calculated as $i_{i v}=-\left(i_{i u}+i_{i w}\right)$. An SP that saves larger current achieves grater $\mathrm{SW}$ loss reduction. For example, when $\left|i_{i u}\right|$ is the largest, the $V_{8}, V_{15}, V_{17} \mathrm{SP}$ or $V_{17}, V_{15}, V_{8} \mathrm{SP}$, which saves the switching of phase $u$ must be selected. Finally, the $\mathrm{SP}$ that is closer to the last SV of the previous switch cycle is selected.

The above technique considers only the SW losses due to PWM switching transitions and does not consider SW loss due to a transition from the previous SP to the present SP. This transition loss must also be considered for optimal minimization of the total loss.

2.3 Total SW Loss Estimation An SW loss is assumed and approximated to be proportional to its current amplitude, and the total loss estimation discussed in this section. The total SW loss for one switch cycle comprises of the transition loss from the last SV in the previous SW cycle to the first SV in the present cycle and the PWM SW loss between the selected SVs in the present cycle. In conventional PWM methods, the PWM SW loss is generated at thrice between the four SVs in any cycle. On the contrary, in the proposed two-phase PWM method, it is generated only twice for one cycle and this can be considered to be a relative loss reduction of one PWM switching transition. Therefore, for convenience, the total SW loss is estimated as the difference between the loss that is reduced using the two-phase modulation and the transition loss.
Table 2. Parameters of the inverter

\begin{tabular}{|l|c|}
\hline parameters & value \\
\hline \hline input dc voltage $E$ & $200 \mathrm{~V}$ \\
\hline frequency of output voltage & $50 \mathrm{~Hz}$ \\
\hline dead time & $2.5 \mu \mathrm{s}$ \\
\hline filter inductance $L$ & $2.78 \mathrm{mH}$ \\
\hline filter capacitance $C$ & $60 \mu \mathrm{F}$ \\
\hline
\end{tabular}

For example, when a desired output voltage vector is in $\mathrm{SM} \mathrm{Ib}$ and the last $\mathrm{SV}$ in the previous switch cycle is $V_{1}$, the initial start $\mathrm{SV}$ must first move from $V_{1}$ to any one of $V_{2}, V_{9}$, $V_{16}, V_{17} \mathrm{SV}$. If selected, the first $\mathrm{SP}$ of $V_{2}, V_{9}, V_{16}$ in the table has a transition loss of $2 i_{i v}$ from $V_{1}$ to $V_{2}$ and a loss saving of $i_{i w}$ from $V_{2}, V_{9}, V_{16}$. Therefore, the total loss estimation is $2 i_{i v}-i_{i w}$. Similarly, the estimations for the other three SPs are $\left(i_{i u}+2 i_{i v}\right)-i_{i v},\left(i_{i u}+i_{i v}\right)-i_{i w}$, and $\left(2 i_{i v}+i_{i w}\right)-i_{i v}$ respectively. The present current amplitudes $i_{i u}, i_{i v}, i_{i w}$ are measured and evaluated in these estimations. Thereafter, the SP with the minimum estimation, which is optimum, and is selected.

The final loss estimation must be slightly modified, considering the weights and characteristics of these two losses. The transition loss is only transient and is generated when there is an SM change. The PWM SW loss is continually generated and it is dominant. In fact, it is often desirable for future switch cycles to reduce steady losses even if some additional loss is necessary in the present cycle. In this study, the weight of the transition loss is somewhat less-weighted with a constant coefficient factor $k<1$ for global optimization.

$$
\begin{aligned}
(\text { total loss })= & (\text { transition loss by SP change }) \times k \\
& -(\text { loss reduction by two phase } \mathrm{PWM})
\end{aligned}
$$

The constant $k$ is set, for example, to be 0.5 . The previous four estimates then become $2 k i_{i v}-i_{i w}, k\left(i_{i u}+2 i_{i v}\right)-i_{i v}$, $k\left(i_{i u}+i_{i v}\right)-i_{i w}, k\left(2 i_{i v}+i_{i w}\right)-i_{i v}$ respectively. This is the proposed optimum PWM principle which is very simple. This loss is estimated for all the possible SPs in Table 1 at the beginning of each SW cycle. The optimum SP, which minimizes this estimation, is then selected, and it effectively reduces the SW loss.

\section{Experimental Verification}

3.1 Two-Phase Modulation Effect of the NPC Inverter

The proposed optimum PWM principle is experimentally verified with a DSP-based control system. It is digitally controlled with a sinusoidal compensator and state feedback gains ${ }^{(14)}$. The PWM output voltages of a three-level NPC inverter connected to a universal load after an LC filter, as shown in Fig. 1, are investigated. The dc input voltage $(E)$ is $200 \mathrm{~V}$, and the ac output voltages are $80 \mathrm{~V}$ in amplitude at $50 \mathrm{~Hz}$. The inverter output voltages are generated in the outer SMs b-d because over-modulations can be avoided. The experimental parameters are listed in Table 2. The SW losses of the inverter are dependent on the switch currents whose output currents are measured and used in for SW loss estimations.

For example, the case in which the load power factor $(\mathrm{PF})=0.5$ with no phase difference between inverter voltages and currents before filtering is shown in Fig. 5(a); here, 

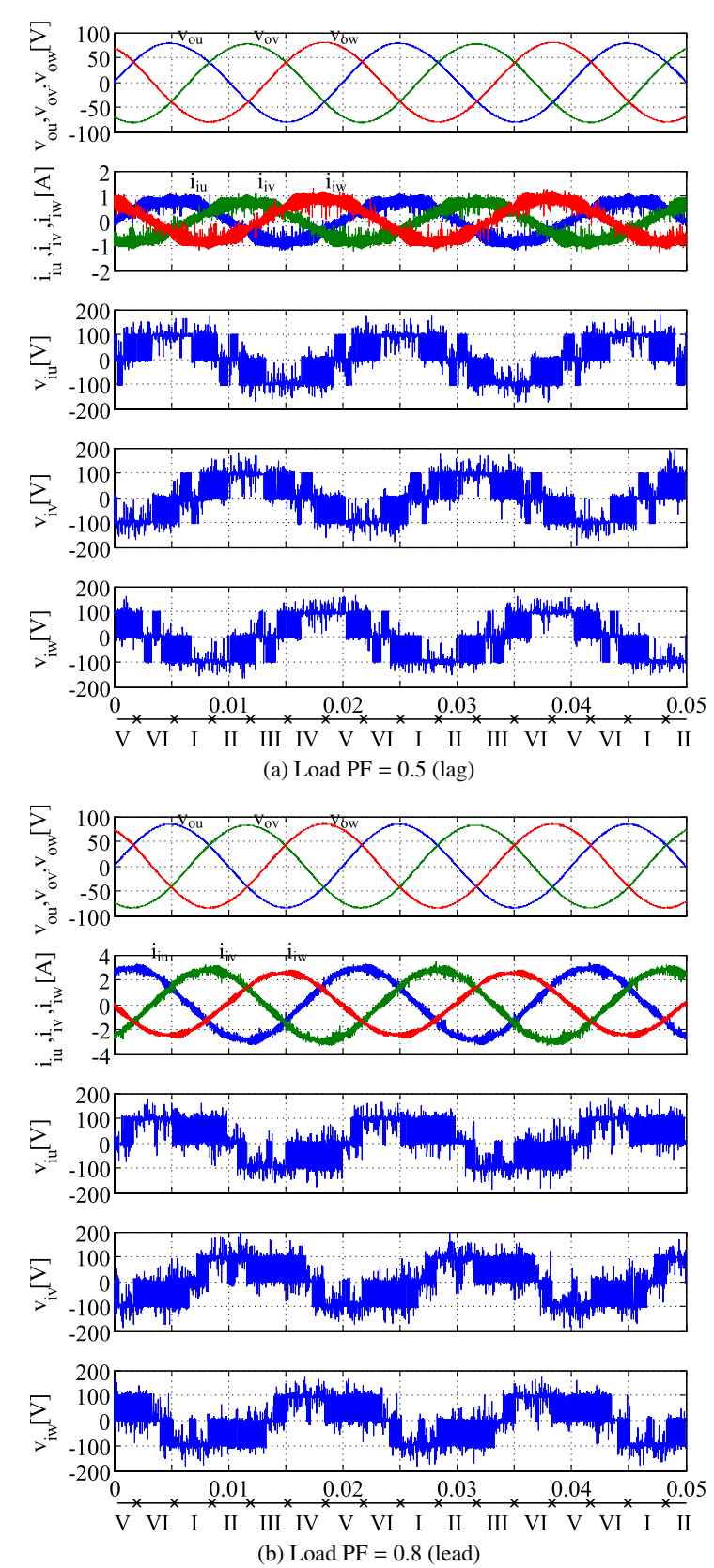

Fig. 5. Output waveforms of the three-level NPC inverter

one unit length of the horizontal axis is $5 \mathrm{~ms}$. The waveforms represent the ac output voltages $\left(v_{o k}\right)$, inverter currents $\left(i_{i k}\right)$, and the PWM voltage waveforms $\left(v_{i k}\right)$ for phases $k=u, v, w$ from the top to the bottom. As can be seen, there are no PWM voltage switching transitions before the current peaks for the time interval of $60^{\circ}$. The interval consists of SMs VIc, VId, Ib, and Ic, where the SPs are $\left(V_{13}, V_{15}\right.$, $\left.V_{25}, V_{15}, V_{13}\right),\left(V_{1}, V_{13}, V_{15}, V_{13}, V_{1}\right),\left(V_{1}, V_{8}, V_{15}, V_{8}, V_{1}\right)$, and $\left(V_{8}, V_{15}, V_{17}, V_{15}, V_{8}\right)$, respectively. The switching of $i_{i u}$ is effectively avoided around at its peak, and number of switching transitions is reduced to $2 / 3$ times. A similar reduction is achieved in other phases. Switching of the largest current is avoided because any phase can be selected.

Another case in which the load PF $=0.8$ is shown in Fig. 5(b) where the inverter currents lead voltages by approximately $40^{\circ}$. There are no PWM switching transitions for the

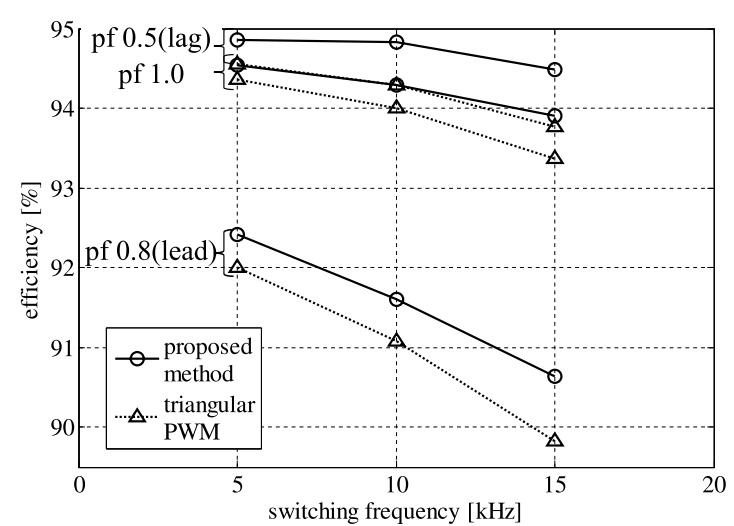

(a) Before filtering

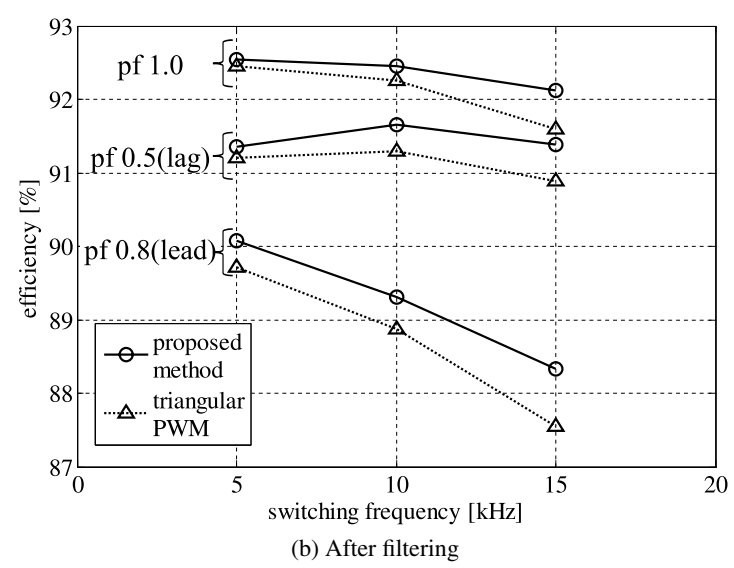

Fig. 6. Efficiencies of the three-level NPC inverter

time interval after the peak of $i_{i u}$ which consists of SM Vc, $\mathrm{Vd}, \mathrm{VIb}$, and VIc where the SPs are $\left(V_{24}, V_{12}, V_{23}, V_{12}, V_{24}\right)$, $\left(V_{24}, V_{12}, V_{6}, V_{12}, V_{24}\right),\left(V_{13}, V_{6}, V_{25}, V_{6}, V_{13}\right)$, and $\left(V_{13}, V_{15}\right.$, $\left.V_{25}, V_{15}, V_{13}\right)$, respectively. Switching of the largest $i_{i u}$ or the secondly largest $i_{i v}$ is effectively avoided and number of switching transitions is reduced to $2 / 3$ times. This reduction is the same as in the other phases.

The proposed method enables the reduction of the number of switching transitions of the largest or the second largest current to $2 / 3$ times. Moreover, it can automatically adapt to different conditions of current amplitudes, which is dependent on the power factors of loads.

\subsection{Comparison of Power Conversion Efficiencies}

Power conversion efficiency of the proposed method is investigated and compared with that of the conventional triangular PWM method. A precision power analyzer WT1800 is used for the measurement. The results of before and after filtering are shown in Fig. 6. Efficiencies are measured for different PFs, 0.5 (lag), 1.0, 0.8 (lead) and for three different carrier frequencies, $5 \mathrm{kHz}, 10 \mathrm{kHz}, 15 \mathrm{kHz}$. The differences between carrier frequencies are considered to be those of switching losses.

The efficiencies of the proposed method are higher than those of the conventional triangular method. The differences are small at $5 \mathrm{kHz}$ because the harmonic contents are slightly increased with the proposed method. However, the difference increases as the switching frequency increases because the SW loss reduction overcomes the increase of harmonic contents in the proposed method. 


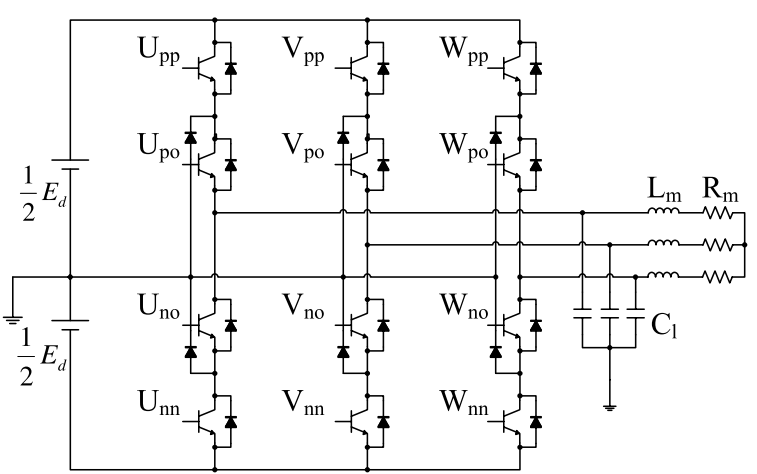

Fig. 7. Three-phase three-level NPC inverter with an inductive load and stray capacitances

\section{Extension of the Proposed Method to Common- Mode Voltage Reduction}

4.1 Common-Mode Voltage Reduction Method The proposed method can effectively reduce switching losses. However, it cannot reduce common-mode voltage and has voltage fluctuations at the neutral point. Therefore, the idea is modified to simultaneously reduce common-mode voltages.

First, the common-mode voltage reduction method in a three-level NPC inverter shown in Fig. 7 is explained briefly. Here, $L_{m}, R_{m}$ and $C_{l}$ denote load inductance, load resistance, and stray capacitance respectively. The phase voltages $V_{u}, V_{v}, V_{w}$ are controlled to satisfy a constraint that $V_{c}=\left(V_{u}+V_{v}+V_{w}\right) / 3$ is zero. The switching losses can be reduced under this zero common-mode voltage condition.

The constraint is satisfied only when the three phase levels are combinations of $\left(1, \frac{1}{2}, 0\right)$ corresponding to the seven SVs from $V_{0}$ to $V_{6}$ in Fig. 8(b) out of the 27 possible vectors in Fig. 8(a). It should be noted that this limitation increases harmonic contents and reduces the maximum ac output voltage. A pair of phases is always switched to satisfy the constraint because one level is replaced with that of another, except in the $V_{0}$ case.

4.2 Reduction of Switching Transitions with the TwoPhase Switching Method In the PWM generation method based on the space-vector concept, first a designated vector is decomposed into three vertex components which surround the vector and are classified as one of the six SMs from I to VI divided by six SVs for $V_{c}=0$ as shown in Fig. 8(b). For example, a vector in SM II shown in Fig. 8 is decomposed into three SVs $V_{0}, V_{1}, V_{2}$ whose ratios correspond to their duties. The original space vector modulation scheme outputs four SVs for one switching cycle. On the contrary, the proposed method outputs only three SVs and reduces the switching transitions from three to two for one switching cycle. Furthermore two phases always switch at the same time and number of voltage fluctuations is reduced. This is expected to reduce the common-mode voltage components even they are not completely eliminated, owing to switching noises

4.3 Selection of Loss Reducing SP Considering Phase Current Amplitudes In the proposed method, there are two sets of switch pairs of phases or four switching transitions in phase level for one switch cycle. Namely, one phase switches twice and the other two switch only once. Three

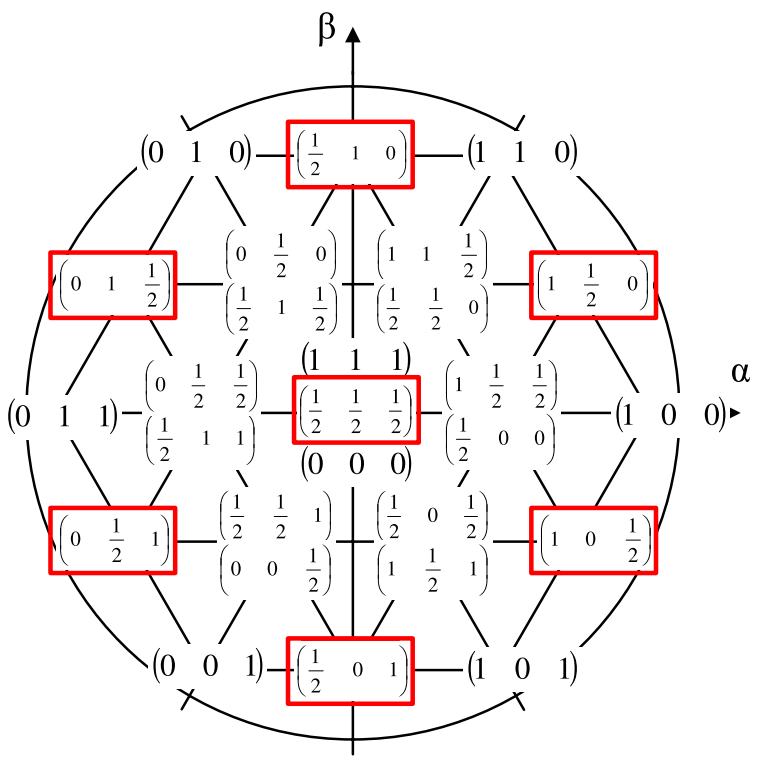

(a) Possible space vectors

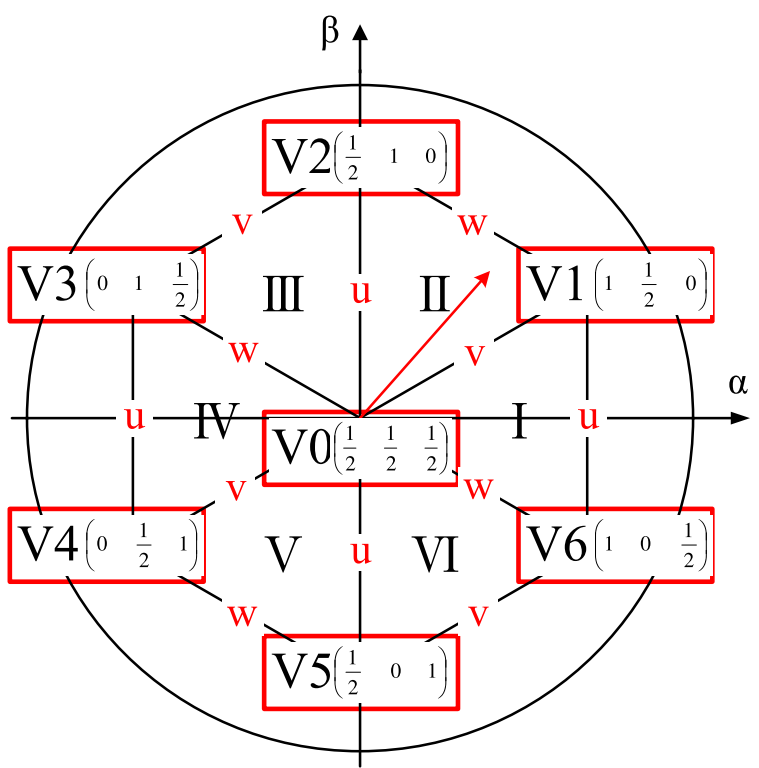

(b) Space vectors selected by the proposed method

Fig. 8. Space-vector diagram of the three-level NPC inverter

possible SPs exist for each SM, and each SP has a different phase, which has two switching transitions for one switch cycle. It is possible to reduce the switching losses by selecting a proper SP, which uniquely switches the minimum current phase twice. Table 3 lists the SPs and their corresponding phases that have two switching transitions. In the table, the double switch phases (d.sw) correspond to the symbols $u, v, w$ in Fig. 8 which shows the phases of two transitions when the switching route is not included in a selected SP. For example, $u$ of SM I on the right-hand side of the table indicates that the SP of $V_{1}-V_{0}-V_{6}$ switches phase $u$ twice. The SP whose $u$ phase current is minimum must be selected. There are three SPs for each SM as shown in Table 3 according to their routes. The SP that has two switching transitions for the smallest current phase and that minimizes the switching losses is uniquely determined on-line by considering the amplitudes of the phases. 
Table 3. Switching patterns for SM I - VI and their phases having double switching transitions

\begin{tabular}{|c|c|c|c|c|c|c|c|c|c|}
\hline I-VI & $V_{a}$ & $V_{b}$ & $V_{c}$ & d.sw & I-VI & & $V_{l}$ & $V_{c}$ & d.sw \\
\hline \multirow[t]{6}{*}{ I } & $V_{6}$ & $V_{0}$ & $V_{1}$ & \multirow[t]{2}{*}{$\mathrm{u}$} & \multirow[t]{6}{*}{ IV } & & $V_{\mathrm{c}}$ & $V_{4}$ & \multirow[t]{2}{*}{$\mathrm{u}$} \\
\hline & $V_{1}$ & $V_{0}$ & $V_{6}$ & & & & $V$ & $V_{3}$ & \\
\hline & $\overline{V_{0}}$ & $\bar{V}_{6}$ & $V_{1}$ & \multirow[t]{2}{*}{$\mathrm{v}$} & & & $\bar{V}$ & $V_{4}$ & \multirow[t]{2}{*}{$\mathrm{v}$} \\
\hline & $V_{1}$ & $V_{\mathrm{G}}$ & $V_{0}$ & & & & $V$ & $V_{0}$ & \\
\hline & $\overline{V_{0}}$ & $\overline{V_{1}}$ & $V_{6}$ & \multirow[t]{2}{*}{ w } & & & $\bar{V}$ & $V_{3}$ & \multirow[t]{2}{*}{ W } \\
\hline & $V_{6}$ & $V_{1}$ & $V_{0}$ & & & & $V$ & $V_{0}$ & \\
\hline \multirow[t]{6}{*}{ II } & $\overline{V_{0}}$ & $\bar{V}_{1}$ & $V_{2}$ & \multirow[t]{2}{*}{$\mathrm{u}$} & \multirow[t]{6}{*}{ V } & & $\bar{V}$ & $V_{5}$ & \multirow[t]{2}{*}{$\mathrm{u}$} \\
\hline & $V_{2}$ & $V_{1}$ & $V_{0}$ & & & & $V$ & $V_{0}$ & \\
\hline & $V_{0}$ & $V_{2}$ & $V_{1}$ & \multirow[t]{2}{*}{$\mathrm{V}$} & & & $\bar{V}$ & $V_{4}$ & \multirow[t]{2}{*}{$\mathrm{V}$} \\
\hline & $V_{1}$ & $V_{2}$ & $V_{0}$ & & & & $V$ & $V_{0}$ & \\
\hline & $V_{1}$ & $V_{0}$ & $V_{2}$ & \multirow[t]{2}{*}{ w } & & & $V$ & $V_{5}$ & \multirow[t]{2}{*}{ w } \\
\hline & $V_{2}$ & $V_{0}$ & $V_{1}$ & & & & V & $V_{4}$ & \\
\hline \multirow[t]{6}{*}{ III } & & $V_{3}$ & $V_{2}$ & \multirow[t]{2}{*}{$\mathrm{u}$} & \multirow[t]{6}{*}{ VI } & & $V$ & $V_{5}$ & \multirow[t]{2}{*}{$\mathrm{u}$} \\
\hline & & $V_{3}$ & $V_{0}$ & & & & $V$ & $V_{0}$ & \\
\hline & & $V_{0}$ & $V_{3}$ & \multirow[t]{2}{*}{$\mathrm{v}$} & & & $V$ & $V_{6}$ & \multirow[t]{2}{*}{$\mathrm{v}$} \\
\hline & $V_{3}$ & $V_{0}$ & $V_{2}$ & & & & V & $V_{5}$ & \\
\hline & & $V_{2}$ & $V_{3}$ & \multirow[t]{2}{*}{ w } & & & $\bar{V}$ & $V_{6}$ & \multirow[t]{2}{*}{ w } \\
\hline & $V_{3}$ & $V$ & $V_{0}$ & & & & $V$ & $V_{0}$ & \\
\hline
\end{tabular}

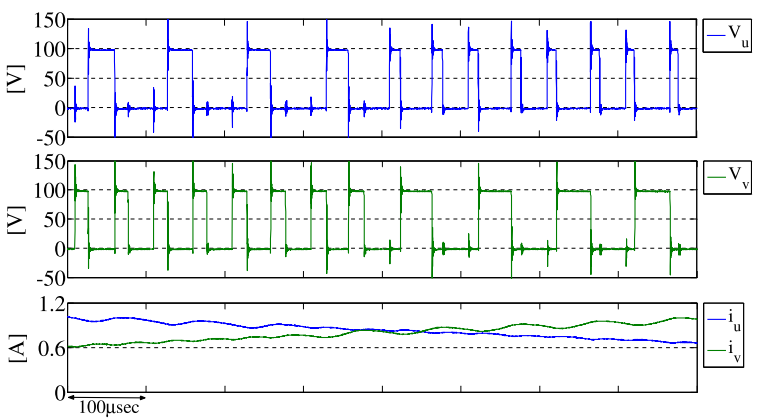

(a) Power factor 1.0

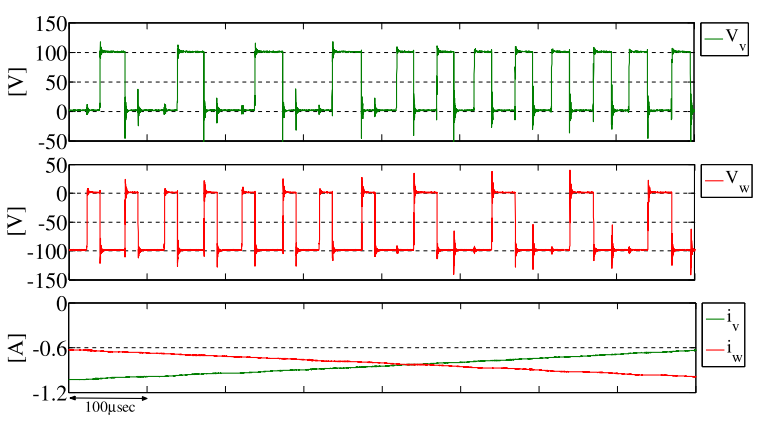

(b) Power factor 0.5

Fig. 9. Output PWM voltage waveforms when the currents of the two phases cross

\section{Experimental Results}

The proposed method outputs appropriate PWM waveforms depending on the selected optimum SP for phase currents in each switch cycle. This is experimentally investigated with a three-level NPC inverter with a parasitic load capacitance $C_{l}=2 \mathrm{nF}$ under $T_{s}=100 \mu \mathrm{s}$ of $10 \mathrm{kHz}$.

The PWM phase voltage and current waveforms for phases $u$ and $v$ are shown in Fig. 9 for a load PF of 1.0. The waveforms are in SM II, and the minimum phase is varied from phase $v$ to phase $u$, as shown in Fig. 9(a). As can be seen on the left-hand side of the figure, the $V_{0}-V_{2}-V_{1}$ SP is selected, and phase $v$ is switched twice for one switching cycle because $i_{i v}$ is minimum. As can be seen on the right-hand side of the figure, the $V_{0}-V_{1}-V_{2}$ SP is selected and the phase $u$ is switched twice for one switching cycle because $i_{i u}$

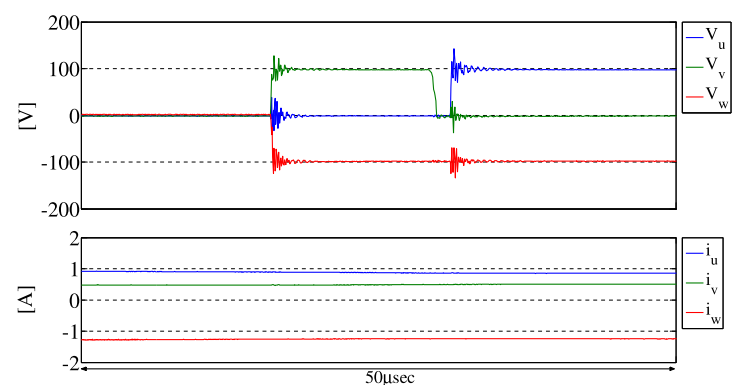

(a) Without dead-time compensation

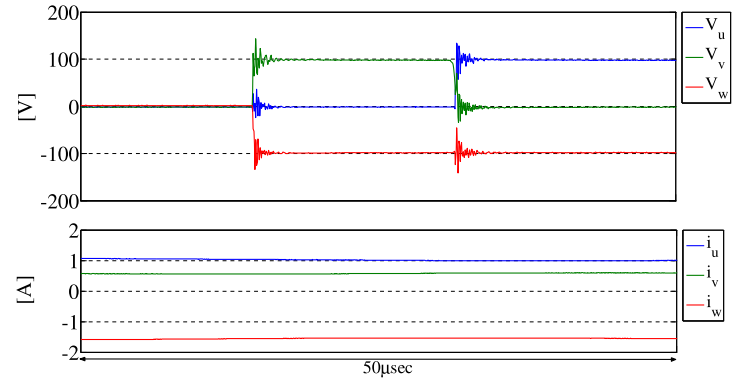

(b) With the proposed dead-time compensation

Fig. 10. Comparison of the effect of dead-time compensation

becomes minimum. For $\mathrm{PF}=0.5$, the waveforms are also in SM II, and the minimum phase is varied from phase $w$ to phase $v$, as shown in Fig. 9(b). As can be seen on the lefthand side of the figure, the $V_{1}-V_{0}-V_{2}$ SP is selected, and the phase $w$ is switched twice because $i_{i w}$ is minimum. The right side of the figure shows that the $V_{0}-V_{2}-V_{1} \mathrm{SP}$ is selected, and phase $v$ is switched twice because $i_{i u}$ becomes minimum. The proposed method selects the optimum SP that minimizes switching losses in any case.

In the proposed method, a pair of phases must be switched synchronously. The output voltages and currents of the SM I I case for $\left(i_{i u}>0, i_{i v}>0, i_{i w}<0\right)$ are measured and shown in Fig. 10(a). A rising timing delay of $V_{v}$ occurs between $V_{2}$ and $V_{1}$ owing to the dead-time effect. After compensation with a timing control scheme, the waveform has no timing delay of $V_{v}$. Both the rising time of $V_{v}$ and the falling time of $V_{w}$ between $V_{0}-V_{2}$ are compensated.

The output voltage and current waveforms of the conventional triangular modulation and proposed methods for a power factor of 1.0 for one cycle are shown in Fig. 11. The waveforms for the phase voltages, common-mode voltages, and phase currents are shown from the top to the bottom. There are fluctuations in the common-mode voltages with the conventional method as can be seen in Fig. 11(a). However, they are reduced, except for the switching noises, with the proposed method, as shown in Fig. 11(b). This is also valid for a power factor of 0.5, as can be seen in Fig. 11(c).

The waveforms are magnified for two symmetric switching cycles, as shown in Fig. 12. The SPs of $V_{0}-V_{1}-V_{2}$ [Fig. 12(b)] and $V_{1}-V_{0}-V_{2}$ [Fig. 12(c)] in SM II minimize the SW losses of phases $u$ and $w$. With the conventional method, the common-mode voltage in Fig. 12(a) varies in steps and it generates leakage currents. With the proposed method, the common-mode voltages shown in Figs. 12(b) and (c) are almost constant except for the switching noises, which are slightly suppressed by snubber capacitors. The proposed 


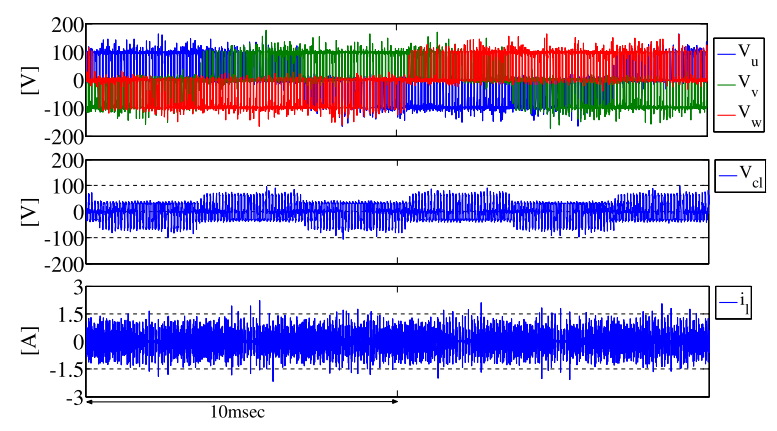

(a) Triangular modulation (power factor 1.0)

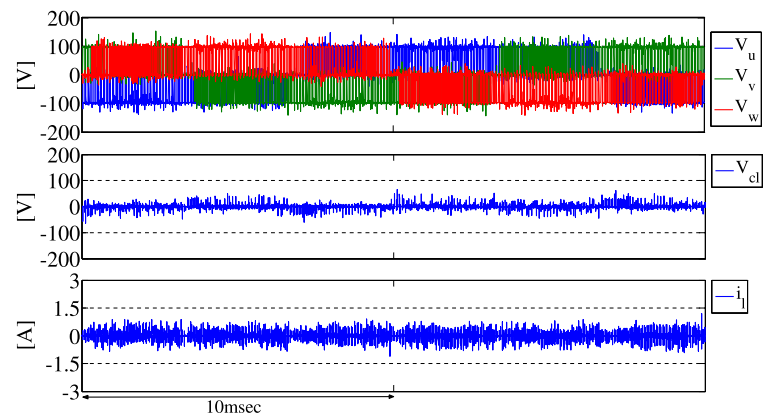

(b) Proposed method (power factor 1.0)

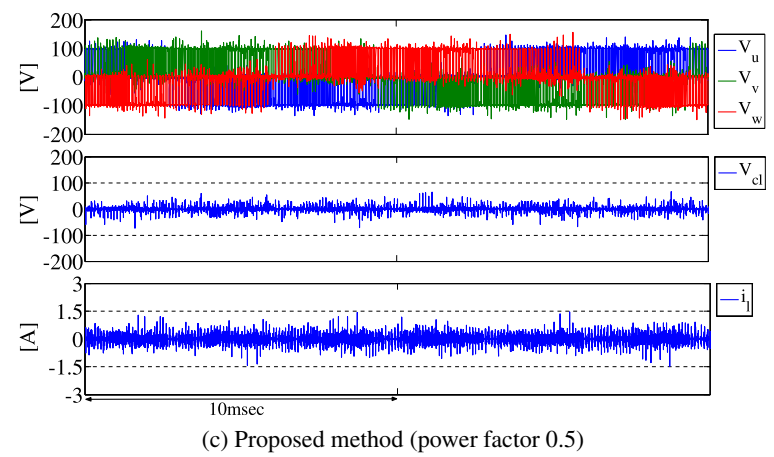

Fig. 11. Output waveforms for one cycle

method can effectively suppress the leakage current. However, further modification is needed for the suppression of switching noises.

Efficiencies of the two methods for PFs of 1.0 and 0.5 are listed in Table 4. The proposed method reduces the number of switching transitions from three to two for one switch cycle. However, it still increases the switching transitions of phases from three to four because it needs a pair of two phases for one switch. The efficiencies of the proposed method are better than those of the simple SV method without the two-phase modulation.

\section{Conclusions}

This study proposed an efficient PWM method that optimally reduces SW losses for one switch cycle using a novel on-line two-phase modulation scheme.

(1) The proposed method optimally selects an SP, which effectively reduces the SW loss, on the basis of the amplitudes of phase currents among the possible SP candidates.

(2) SW losses are estimated for all possible SVs by subtracting the SW loss savings obtained with the twophase modulation from $k$ times the transition losses.

(3) The proposed method can automatically adapt to

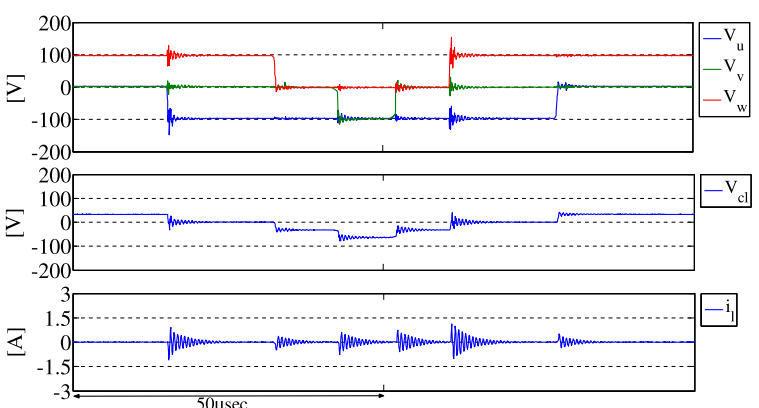

(a) Triangular modulation (power factor 1.0)

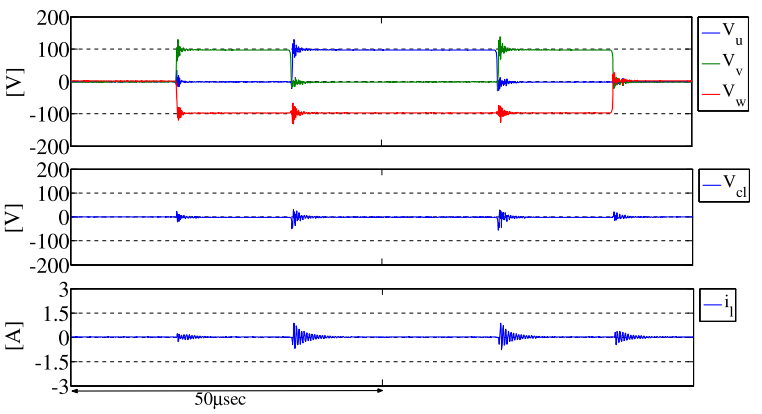

(b) Proposed method (power factor 1.0)

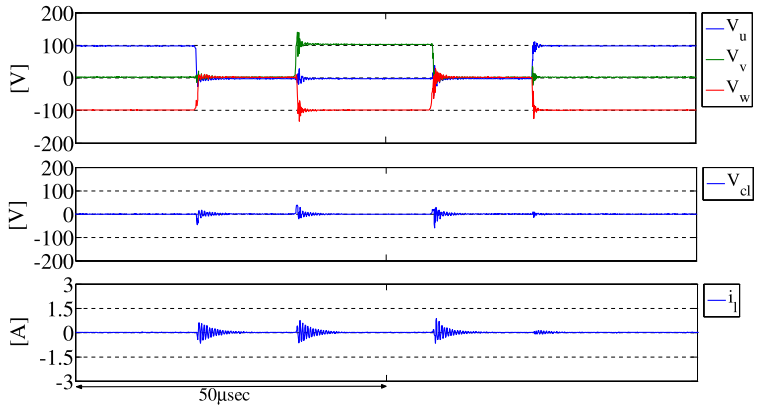

(c) Proposed method (power factor 0.5)

Fig. 12. Output waveforms for two symmetric switching cycles

Table 4. Comparison of efficiencies of different methods

\begin{tabular}{|c|c|c|}
\hline \multirow{2}{*}{ Control methods } & \multicolumn{2}{|c|}{ Efficiency } \\
\cline { 2 - 3 } & PF 0.5 & PF 1.0 \\
\hline Conventional SV modulation & $87.4 \%$ & $94.1 \%$ \\
\hline Proposed method & $88.7 \%$ & $95.0 \%$ \\
\hline
\end{tabular}

different load conditions.

(4) The proposed method is applicable to both off-line and on-line PWM controls.

(5) It reduces number of switching transitions of the largest or second largest current to approximately $2 / 3$ times. The proposed method is validated for its $\mathrm{SW}$ loss reduction capability.

(6) The proposed method can be extended to commonmode voltage reduction. The principle is to eliminate the common-mode voltage by limiting the combination of output phase voltage patterns of the three-level NPC inverter. This limitation increases the harmonic contents and reduces the maximum output ac voltage. The proposed method is validated for its ability to effectively reduce common-mode voltages and switching losses. The efficiency can be enhanced, for example, by suppressing the switching noises. An 
investigation on appropriate snubbers is needed in future work.

\section{References}

( 1 ) K. Taniguchi and H. Irie: "Trapezoidal modulating signal for three-phase PWM inverter", IEEE Trans. on Ind. Electron., Vol.33, No.2, pp.193-200 (1986)

( 2 ) S. Halasz, B.T. Huu, and A. Zakharov: "Two-phase modulation technique for three-level inverter-fed AC drives", IEEE Trans. on Ind. Electron., Vol.47, No.6, pp.1200-1211 (2000)

( 3 ) M.C. Cavalcanti, E.R.C. da Silva, A.M.N Lima, C.B. Jacobina, and R.N.C. Alves: "Reducing losses in three-phase PWM pulsed DC-link voltage-type inverter systems", IEEE Trans. on Ind. Appl., Vol.38, No.4, pp.1114-1122 (2002)

( 4 ) H. Lu, W. Qu, X. Cheng, Y. Fan, and X. Zhang: "A Novel PWM Technique With Two-Phase Modulation", IEEE Trans. on Power Electronics, Vol.22, No.6, pp.2403-2409 (2007)

( 5 ) B. Kaku, I. Miyashita, and S. Sone; "Switching loss minimised space vector PWM method for IGBT three-level inverter", IEE Proc.-Electr.Power Appl., Vol.144, No.3, pp.182-190 (1997)

( 6 ) Y.S. Lai and R. Bowes; "Optimal bus-clamped PWM techniques for threephase motor drives", Proc. of the IEEE IECON Conference (2004)

( 7 ) H. Fujita and R. Suzuki; "A three-phase solar power conditioner using a single-phase PWM control method", IEEJ Trans. IA, Vol.130, No.2, pp.173180 (2010)

( 8 ) S. Motegi, S. Masukawa, and Y. Nishida: "Novel two-phase PWM scheme for three-phase voltage-fed converter", IEEJ Tras. IA, Vol.132, No.9, pp.940$941(2012)$

( 9 ) T. Kato, K. Inoue, Y. Taniyama, and K. Yamada: "Optimum reduction of switching losses based on the two-phase PWM modulation method for twolevel inverter", 2012 IEEE 13th Workshop on Control and Modeling for Power Electronics (COMPEL), pp.1-6 (2012)

(10) Z. Erkuan and T.A. Lipo: "Improvements in EMC performance of inverterfed motor drives", IEEE Trans. on Ind. Appl., Vol.31, No.6, pp.1247-1256 (1995)

(11) K.R.M.N. Ratnayake and Y. Murai: "A novel PWM scheme to eliminate common-mode voltage in three-level voltage source inverter", PESC98 Record, IEEE, Vol.1, pp.269-274 (1998)

(12) H. Zhang, A.V. Jouanne, S. Dai, A.K. Wallace, and F. Wang: "Multilevel inverter modulation schemes to eliminate common-mode voltage", IEEE Trans. on Ind. Appl., Vol.36, pp.1645-1653 (2000)

(13) H.-J. Kim, H. Dong, and S.-K. Sul: "A new PWM strategy for common mode voltage reduction in neutral-point clamped inverter-fed AC motor drives", IEEE Trans. on Ind. Appl., Vol.37, pp.1840-1845 (2001)

(14) T. Kato, K. Inoue, and S. Kuroda: "Sinusoidal waveform following method for digital control of PWM inverter", IEEJ Trans. IA, Vol.126, No.3, pp.218224 (2006)

Toshiji Kato (Senior Member) was born in Kyoto, Japan. He received

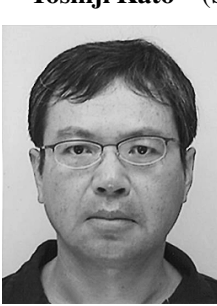
his B.E., M.E., and Ph.D. degrees from Doshisha University, Kyoto, Japan, in 1979, 1981, and 1986 respectively. Since 1981, he has been with Doshisha University, where he is now a Professor in the Department of Electrical Engineering. He was a visiting scientist of IREQ from April to August of 1990 and of MIT LEES from 1990 to 1992 . His primary interests include computer analysis and control of power and power electronic systems. He is a member of the IEEE, CIGRE, and IEE of Japan.
Kaoru Inoue (Member) was born in Osaka, Japan. He received B.E.

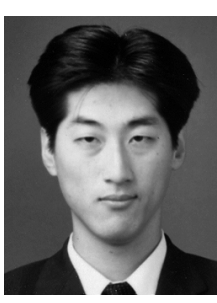
and M.E. degrees from Kansai University in 1996 and 1998, respectively. He also received Ph.D. degree from Osaka University in 2001. He was a Research Fellow of the Japan Society for the Promotion of Science from 2000 to 2001. Since 2001, he has been with Department of Electrical Engineering, Doshisha University, where he is now a Professor. He was a visiting scholar of the Department of Electrical Engineering and Computer Science (EECS) at the University of California, Berkeley from 2007 to 2008. His research interest includes analysis and control of power electronic and motor drive systems. He is a member of the IEEE, IEICE, and IEE of Japan.

Kyu Takano (Non-member) was born in Fukui, Japan. He received

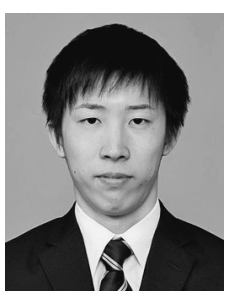
B.E., and M.E. degrees from Doshisha University, Kyoto, Japan, in 2011 and 2013, respectively. He joined Shimadzu Corporation in 2013. His research interests include EMC control techniques.

Kazunari Yamada (Student Member) was born in Nagoya, Japan. He

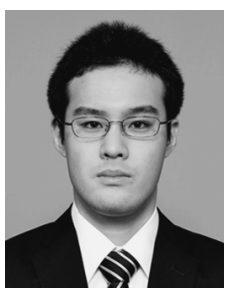
eceived B.E. and M.E. degrees from Doshisha University, Kyoto, Japan, in 2012 and 2014, respectively. He joined Nippon Sharyo, Ltd. in 2014. His reaserch interests include inverter control techniques.

Ariyasu Aki (Non-member) was born in Osaka, Japan. He received

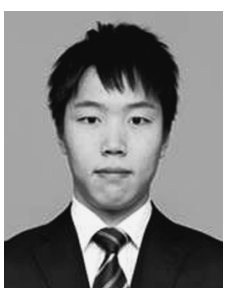
B.E. degree from Doshisha University, Kyoto, Japan, in 2013. He is currently a Master's degree student at Doshisha University. His research interests are inverters and EMC control techniques. 